\title{
Increased automatic spreading of activation in thought-disordered schizophrenic patients
}

\author{
Steffen Moritz ${ }^{\mathrm{a}, *, 1}$, Todd S. Woodward ${ }^{\mathrm{b}}$, Daniela Küppers ${ }^{\mathrm{a}}$, Alexandra Lausen ${ }^{\mathrm{a}}$, \\ Marc Schickel $^{\mathrm{a}}$ \\ ${ }^{a}$ University Hospital of Hamburg, Hospital for Psychiatry and Psychotherapy, Martinistrasse 52, 20246 Hamburg, Germany \\ ${ }^{\mathrm{b}}$ Department of Medicine and Research, Riverview Hospital, 500 Lougheed Highway, Port Coquitlam, B.C., Canada V3C $4 J 2$
}

Received 20 May 2001; accepted 29 August 2001

\begin{abstract}
Previous research on semantic priming in schizophrenia has produced conflicting findings. While several studies provided evidence for an enhanced cognitive spreading of activation in schizophrenia patients with formal thought disorder, other research has failed to replicate. The aim of the present study was to resolve some of the ambiguities in the literature. Thirty-two schizophrenic patients (12 with and 20 without symptoms of formal thought disorder according to the PANADSS) and 65 healthy controls were compared in a semantic priming task using word pronunciation. Irrespective of baseline condition (neutral or unrelated condition) patients with formal thought disorder (TD) exhibited a significantly greater indirect semantic priming effect than both non-TD patients and healthy controls. Known confounding variables such as length of illness, neuroleptic dosage and psychomotor slowness did not moderate priming. Results further strengthen the spreading activation model of formal thought disorder put forward by Maher/Manschreck and Spitzer. Data indicate that hyper-priming is not confined to lexical decision tasks. Possible reasons why several studies have failed to replicate greater priming in TD schizophrenic patients are discussed.
\end{abstract}

(C) 2002 Elsevier Science B.V. All rights reserved.

Keywords: Semantic priming; Schizophrenia; Formal thought disorder; Language

\section{Introduction}

Using the semantic priming paradigm, a technique adopted from cognitive psychology, Maher et al. (1987) and Spitzer et al. (1993a, 1994) have provided empirical evidence for the hypothesis 3that enhanced spreading of activation underlies positive

\footnotetext{
${ }^{*}$ Corresponding author.

E-mail addresses: smoritz@cortex.psych.ubc.ca, moritz@uke.uni-hamburg.de (S. Moritz).

${ }^{1}$ Tel.: +49-40-42803-6565; fax: +49-40-42803-5121.
}

formal thought disorder (associative loosening/tangential speech) in schizophrenia. For semantic priming experiments, trials each consisting of two strings of characters/words (referred to as prime and probe) are successively presented. The subject is usually instructed to silently attend to the first string of characters/word and then either (a) has to read aloud the probe [word pronunciation (WP) procedure] or (b) has to decide whether the probe is a word or a string of meaningless characters [lexical decision (LD) procedure]. Instructional emphasis is put on response speed. 
A large literature from cognitive psychology shows that responses are reliably facilitated to a probe that is preceded by a semantically related prime word (e.g. sister-brother) relative to probes which are preceded by unrelated (e.g. hat-table) or neutral primes (e.g. $\mathrm{xxx}$-table). This so-called semantic priming effect is attributed to a spread of activation initiated by the display of the prime word which is thought to pre-activate semantically related information (Neely, 1977, 1991). Whereas stimulus onset asynchronies (SOA; interval between prime and probe onset) below 400/ $500 \mathrm{~ms}$ solely reflect automatic spreading of activation, priming effects for larger SOAs may also be influenced by expectancy and other controlled processes.

Maher et al. (1987), Spitzer et al. (1993a) (for a review, see Spitzer, 1997) and, more recently, Weisbrod et al. (1998) were able to demonstrate that TD schizophrenic patients exhibit increased semantic priming at short SOAs. It is argued that these patients display a greater automatic spreading of activation in the semantic network. Spitzer measured even greater priming effects in TD patients when using indirectly related words (i.e. words which are indirectly mediated through a nonpresented word, tiger-lion-stripes, day-night-black). This suggests that TD schizophrenic patients do not only have faster but also farther-reaching associations than controls.

Despite this set of findings, the literature on semantic priming has remained contradictory, since several studies have not found larger priming in schizophrenia (Barch et al., 1996; Chapin et al., 1989, 1992; Ober et al., 1995; Vinogradov et al., 1992). This failure to find enhanced semantic priming in schizophrenia may be accounted for by several methodological shortcomings in these studies (see also Moritz et al., 1999 for more details).

(a) Several studies have used very short prime presentation times (lower or equal to $100 \mathrm{~ms}$; Barch et al., 1996; Blum and Freides, 1995; Ober et al., 1995; Vinogradov et al., 1992) that may preclude deep semantic processing in schizophrenic patients since most patients are perceptually slowed and have problems perceiving quickly presented material (see Cadenhead et al., 1997).

(b) Some studies did not subdivide patients according to the presence of formal thought disorder (Chapin et al., 1989, 1992; Ober et al., 1995, 1997; Vinogra- dov et al., 1992). Enhanced priming is not inferred for all syndromatic patterns of schizophrenia but only for positive formal thought disorder. Therefore, these studies do not falsify the initial claims made by Maher et al. (1987).

(c) Some studies have used rather long SOAs ( $\geq 500 \mathrm{~ms}$ ) which do not exclusively tap automatic processes (e.g. Aloia et al., 1998) and, thus, do not challenge the basic hypothesis.

(d) All studies that failed to replicate Maher's results have measured direct semantic priming effects. However, according to the theoretical account put forward by Spitzer (1997), the assessment of indirect semantic priming is a more valid and powerful correlate of enhanced spreading of activation, since it allows estimation of how fast and how far associations spread in semantic networks (Spitzer, 1997).

On the other hand, methodological concerns have also been raised against the original studies conducted by Maher et al. (1987) (also Manschreck et al., 1988) and Spitzer et al. (1993a,b). Barch et al. (1996) have pointed out that greater than normal priming has only been demonstrated in LD tasks but not in WP tasks. They argue that LD tasks may not solely measure automatic processes. Moreover, it has been suggested that the computation of priming ratios as carried out by Spitzer might not adequately address the impact of psychomotor slowing on priming. Finally, it has been claimed that the effects of antipsychotic medication were not sufficiently addressed.

In three previous studies, we have collected additional evidence in support of the hyper-priming hypothesis while addressing several of the aforementioned objections. In our first study (Moritz et al., 1999), we split a large sample of healthy subjects $(n=156)$ according to a measure of language disturbance (Frankfurt Complaint Questionnaire subscale language). We found significantly greater priming in subjects with elevated language disturbance scores. Importantly, we were able to show that priming for short SOAs $(200 \mathrm{~ms})$ was not associated with psychomotor slowing. This suggests that motor retardation as evidenced by overall greater RT latencies in schizophrenic patients alone does not imply elevated priming. In a second study (Moritz et al., 2001a), we have replicated Spitzer et al.'s (1993a) results using an identical priming paradigm. TD schizophrenic patients showed greater priming than psychiatric and 
healthy controls. Neuroleptic dosage did not correlate with priming. In our most recent study (Moritz et al., 2001b), we used a WP approach and successfully demonstrated that enhanced semantic priming in TD schizophrenia is not confined to LD tasks. An additional result was that associations of TD schizophrenic patients did not only spread further but were also more oblique: patients showed significantly stronger priming for probe words that were inferior associates of a homograph prime category (e.g. bank-river) whereas no difference occurred for dominant probes (e.g. bank-money). Again, psychomotor slowing and neuroleptic dosage were not associated with strength of priming (moreover, both subgroups did not differ regarding neuroleptic treatment). However, this study did not employ an indirect semantic priming condition.

The present study addresses two major issues: (a) this is the first study which investigates indirect semantic priming in TD schizophrenic patients using a WP task, (b) two different baseline conditions were assessed in an attempt to demonstrate that enhanced priming does not depend upon baseline condition used (neutral or unrelated). Our position is that the spreading activation hypothesis of formal thought disorder will be substantially strengthened if hyperpriming is demonstrated with different experimental procedures (WP and LD, different baseline conditions).

\section{Methods}

\subsection{Subjects}

For the present study, 32 psychiatric inpatients fulfilling DSM-IV criteria of schizophrenia were recruited [age: 32.53 (S.D.: 8.75); 9 women, 23 men; education: 11.37 years (S.D.: 2.19), length of illness: 4.85 years (S.D.: 6.31), chlorpromazine equivalent dosage: $389.69 \mathrm{mg}$ (202.53)]. Patients were on atypical neuroleptic agents for at least 2 weeks and no further antidepressive or antiparkinson medication was administered. Diagnoses were confirmed using a semi-structured interview. None of the subjects participated in one of our previous studies on semantic priming (see Moritz et al., 1999, 2001a,b). The sample was divided according to the presence of associative loosening which was rated according to the Positive and Negative and Disorganized Symptoms Scale (PANADSS; Andresen and Moritz, 2000). The PANADSS is a psychopathological rating system whose scores rely on anchor points for all rating degrees (17) to enhance reliability. Twelve subjects exhibited at least mild forms of associative loosening (score $\geq 3$ ). Preliminary analysis shows excellent interrater reliability for the associative loosening item $(r=0.92)$. Psychopathology ratings were performed without knowledge of task performance. Other symptoms were rated using the Positive and Negative Syndrome Scale (PANSS; Kay et al., 1989). The PANSS rating for formal thought disorder was considered insufficient for our purposes since it confounds various positive (associative loosening) and negative symptoms (mutism, blocking of speech) of formal thought disorder.

Sixty-five subjects served as healthy controls [age: 34.20 (S.D.: 11.32); 28 women, 37 men; education: 11.57 years (S.D.: 1.68)]. All subjects were German and had no history of significant brain pathology (e.g. stroke, MS, brain operations, epilepsy) or drug abuse. All patients gave informed consent to participate.

\subsection{Task}

The experiment was performed on Macintosh computers. Trials were preceded by a fixation point for $700 \mathrm{~ms}$. Prime words were displayed for $200 \mathrm{~ms}$ immediately followed by a probe word $(\mathrm{SOA}=200$ $\mathrm{ms}$ ). Subjects were instructed to silently read the first word and to read aloud the probe word as quickly and as accurately as possible with an emphasis on speed. The display was terminated when subjects triggered the voice key. All stimuli were German words with no word occurring twice. Subsequent trials were initiated by the experimenter via mouse-click. Voice onset latencies were automatically recorded by computer.

The following conditions were presented (trials followed in random order):

(a) Indirect semantic priming condition (e.g. stonesoft, 20 pairs)

(b) semantic priming condition (e.g. sun-moon, 20 pairs)

(c) unrelated condition (e.g. shelter-paint, 20 pairs; no direct association between prime and probe) 
(d) neutral condition (e.g. $\mathrm{xxx}$-ear, 20 pairs, the prime is a string of " $\mathrm{x}$ " which is followed by a regular word).

Word pairs for these conditions were taken from Spitzer et al. (1994) and McNamara and Altarriba (1988). Words across conditions were comparable regarding length and word frequency. The semantic priming effect was computed as follows: median reaction time of the priming condition (semantic or indirect semantic condition) minus the median reaction time of the baseline condition (unrelated or neutral condition). Thus, priming effects were expressed as negative values.

\section{Results}

\subsection{Background variables}

Patients and controls did not differ on verbal intelligence (measured by a vocabulary test, $t=1.24$; $P>0.2)$, age $(t=0.73$; NS), years of education ( $t=$ 0.45 ; NS) and gender $\left(\chi^{2}=2.03\right.$; NS $)$. When the schizophrenic sample was split according to the presence of formal thought disorder ( $n=12$ with TD) again no differences occurred regarding verbal intelligence $(P>0.4)$, age $(P>0.7)$, years of education $(P>0.6)$ and gender $(P>0.2)$. Both schizophrenic subgroups did not differ regarding number of hospitalizations [TD: 4.09 (5.56); NTD: 2.53 (2.46); $P>0.2$ ], length of illness [TD: 7.27 years (8.06); NTD: 3.52 years
(4.84); $P>0.1]$ and neuroleptic dosage [chlorpromazine equivalents; TD: $330.56 \mathrm{mg}$ (184.47); NTD: $321.76 \mathrm{mg}$ (200.84); $P>0.9$ ].

\subsection{Priming}

A two-way repeated measures analysis of variance (ANOVA) with group (healthy controls, TD, NTD) as between-subject and experimental conditions (neutral, unrelated, semantic, indirect semantic) as within-subject variables revealed a significant effect for group $[F(2,94)=18.79 ; P \leq 0.001]$, condition $[F(3,282)=21.84 ; P \leq 0.001]$ and the group$\times$ condition interaction $[F(6,282)=4.32 ; P \leq 0.001]$. The significant main effects reflect prolonged reaction times in both schizophrenic subgroups and reaction time differences for the four conditions (i.e. facilitation effects in the priming conditions relative to both control conditions). The source for the significant interaction was further inspected conducting oneway ANOVAs with priming effects as dependent variables.

For the semantic priming effect where the unrelated condition was used as baseline, no group differences occurred. However, when the neutral condition served as baseline TD patients exhibited significantly stronger semantic priming. For indirect semantic priming as measured using both the neutral and the unrelated baseline condition, the TD-group displayed significantly greater priming effects (see Table 1). In addition, Table 1 shows which priming effects achieved significance for the different groups (paired $t$-tests).

Table 1

Mean reaction times (ms) and standard deviations for the priming effects

\begin{tabular}{lcccc}
\hline & NTD $(n=20)$ & $\mathrm{TD}(n=12)$ & $\mathrm{H}(n=65)$ & ANOVA post hocs (FLSD) \\
\hline Neutral baseline & & & \\
Direct semantic priming & $-18.38(30.71)^{*}$ & $-65.00(86.85)^{*}$ & $-21.84(24.88)^{*}$ & $F=8.86 ; P=0.002 \mathrm{TD}<\mathrm{H}, \mathrm{NTD}$ \\
Indirect semantic priming & $-4.93(32.18)$ & $-56.67(82.38)^{*}$ & $-9.33(34.82)^{*}$ & $F=6.91 ; P=0.002 \mathrm{TD}<\mathrm{H}, \mathrm{NTD}$ \\
& & & & \\
Unrelated baseline & & & & \\
Direct semantic priming & $-9.53(25.80)$ & $-24.33(43.95)^{*}$ & $-13.38(22.45)^{*}$ & $F=1.21 ; P>0.3 \mathrm{NS}$ \\
Indirect semantic priming & $3.93(29.16)$ & $-16.00(26.21)^{*}$ & $-0.87(20.19)$ & $F=2.95 ; P=0.057 \mathrm{TD}<\mathrm{H}, \mathrm{NTD}$ \\
\hline
\end{tabular}

Priming effects were computed as follows: RT experimental condition - RT baseline condition. Post hoc tests were computed with Fisher's LSD (FLSD; $P \leq 0.05$, two-tailed). $\mathrm{H}=$ healthy controls, $\mathrm{NTD}=$ non-thought-disordered schizophrenic patients, $\mathrm{TD}=$ thought-disordered schizophrenic patients.

* Paired $t$-test between experimental and baseline condition, $P \leq 0.05$, one-tailed. 


\subsection{Impact of psychomotor slowing on priming}

Since slowing might inflate priming effects, Chapman et al. (1994) recommended an assessment of the relationship between motor retardation and priming. Following their method, a linear regression analysis was carried out with sum median reaction time of all conditions as independent variable and priming effects as dependent variables. This revealed that slow performance is associated with decreased rather than with increased priming effects, at least for the unrelated conditions (indirect semantic-neutral baseline: $R^{2}=0.00$, NS; semantic-neutral baseline: $R^{2}=-0.01$, NS; indirect semantic-unrelated baseline: $R^{2}=0.04$, $P<0.05$; semantic-unrelated baseline: $R^{2}=0.09$, $P<0.01$ ).

\subsection{Impact of psychopathological and sociodemo- graphic variables on priming}

Length of illness, gender, age, number of hospitalizations, premorbid intelligence, and years of education were unrelated to each of the four priming difference scores $(|r|<0.26$; not significant after adjustment for false-positives). With respect to medication, chlorpromazine equivalents were not correlated with any priming effect $(r=-0.12$ to -0.17 ; $P>0.2)$. Moreover, when the schizophrenic group was split according to the presence of hallucinations, feelings of persecution and the median PANSS global score [65; mean: 65.44 (20.48)] no significant differences emerged (at least $P>0.1$; symptom scores were derived from the PANSS positive subscale; i.e. items 1 and 6).

\section{Discussion}

The present study confirms previous data collected by Maher and Manschreck (Maher et al., 1987; Manschreck et al., 1988), Spitzer et al. (1993a,b), Weisbrod et al., 1998) and our group (Moritz et al., 1999, $2001 \mathrm{a}, \mathrm{b})$ that excessive automatic spreading of activation in the semantic network of schizophrenic patients may be a major contributor to the manifestation of formal thought disorder. The assessment of formal thought disorder was based on a reliable item (interrater reliability: $r=0.92$ ) derived from the PANADSS, a newly developed scale for measuring schizophrenic psychopathology (Andresen and Moritz, 2000).

The present study ruled out concerns raised by Barch et al. (1996) and others regarding previous studies reporting hyper-priming in TD patients. First, the study replicates our findings that increased priming in TD schizophrenic patients can be demonstrated with both LD and WP tasks (Moritz et al., 2001a,b). Secondly, we have repeatedly demonstrated that psychomotor slowing and neuroleptic dosage do not necessarily enhance priming, at least for short SOAs (i.e., the ratio procedure adopted by Spitzer may have over-compensated the effects of psychomotor slowing making conclusions even more conservative). Moreover, this is the first study to demonstrate that enhanced priming does not depend on the baseline conditions used (unrelated/neutral condition).

An interesting question for future research is to reveal whether enhanced priming is exclusively a correlate of formal thought disorder in schizophrenia, or also underlies formal thought disorder in other psychiatric illnesses, most importantly mania. It would be important to inspect whether flight of ideas commonly found in mania is governed by the same putative cognitive mechanism as derailed thinking/ associative loosening in schizophrenia. A study conducted by Kwapil et al. (1990) has already studied bipolar patients, but unfortunately no data was presented to the extent of manic language disturbances in that group.

In addition, more research must be directed towards diagnostic assessment of TD. Until today, TD is often mistakenly allocated to the positive syndrome (both the commonly administered PANSS and the SAPS subsume formal thought disorder under positive symptomatology according to the conventional algorithm) although an extensive factor analytic literature clearly demonstrates that TD, together with inadequate affect and bizarre behaviour, constitutes a separate psychopathological dimension referred to as disorganisation (e.g. Liddle, 1987; Moritz et al., in press). Additional evidence for the separation of formal thought disorder from positive symptomatology is that formal thought disorder is caused by hypo- rather than hyper-dopaminergity (for a review, see Salomé et al., 2000).

As has been discussed more thoroughly elsewhere (Moritz et al., 1999) the failure of several studies to find enhanced priming in TD is likely attributable to 
the following methodological flaws: small sample size, no division of samples according to the presence of TD, briefly presented primes and usage of semantic priming which is considered a less valid correlate of automatic spreading activation than indirect semantic priming (see Spitzer, 1997).

\section{References}

Aloia, M.S., Gourovitch, M.L., Missar, D., Pickar, D., Weinberger, D.R., Goldberg, T.E., 1998. Cognitive substrates of thought disorder: II. Specifying a candidate cognitive mechanism. Am. J. Psychiatry 155, 1677-1684.

Andresen, B., Moritz, S., 2000. Positive and Negative and Disorganized Symptoms Scales for Schizophrenia (PANADSS). Manual. PPV, Westerau.

Barch, D.M., Cohen, J.D., Servan-Schreiber, D., Steingard, S., Steinhauer, S.R., van Kammen, D.P., 1996. Semantic priming in schizophrenia: an examination of spreading activation using word pronunciation and multiple SOAs. J. Abnorm. Psychol. $105,592-601$.

Blum, N.A., Freides, D., 1995. Investigating thought disorder in schizophrenia with the lexical decision task. Schizophr. Res. 16, 217-224.

Cadenhead, K.S., Geyer, M.A., Butler, R.W., Perry, W., Sprock, J., Braff, D.L., 1997. Information processing deficits of schizophrenia patients: relationship to clinical ratings, gender and medication status. Schizophr. Res. 28, 51-62.

Chapin, K., Vann, L.E., Lycaki, H., Josef, N., Meyendorff, E., 1989. Investigation of the semantic network in schizophrenia using the semantic priming paradigm. Schizophr. Res. 2, 355-360.

Chapin, K., McCown, J., Vann, L., Kenney, D., Youssef, I., 1992. Activation and facilitation in the lexicon of schizophrenics. Schizophr. Res. 6, 251-255.

Chapman, L.J., Chapman, J.P., Curran, T.E., Miller, M.B., 1994. Do children and the elderly show heightened semantic priming? How to answer the question. Dev. Rev. 14, 159-185.

Kay, S.R., Opler, L.A., Lindenmayer, J.-P., 1989. The positive and negative syndrome scale (PANSS). Rationale and standardisation. Br. J. Psychiatry 155 (Suppl. 7), 59-65.

Kwapil, T.R., Hegley, D.C., Chapman, L.J., Chapman, J.P., 1990. Facilitation of word recognition by semantic priming in schizophrenia. J. Abnorm. Psychol. 99, 215-221.

Liddle, P.F., 1987. Schizophrenic syndromes, cognitive performance and neurological dysfunction. Psychol. Med. 17, 49-57.

Maher, B., Manschreck, T.C., Hoover, T.M., Weisstein, C.C., 1987. Thought disorder and measured features of language production. In: Harvey, P.D., Walker, E.F. (Eds.), Positive and Negative Symptoms in Psychosis. Erlbaum Associates, Hillsdale, NJ, pp. 195-215.

Manschreck, T.C., Maher, B.A., Milavetz, D.A., Weisstein, C.C., Schneyer, M.L., 1988. Semantic priming in thought disordered schizophrenic patients. Schizophr. Res. 1, 61-66.
McNamara, T., Altarriba, J., 1988. Depth of spreading activation revisited: semantic mediated priming occurs in lexical decisions. J. Mem. Lang. 27, 545-559.

Moritz, S., Andresen, B., Domin, F., Martin, T., Probsthein, E., Kretschmer, G., Krausz, M., Naber, D., Spitzer, M., 1999. Increased automatic spreading activation in healthy subjects with elevated scores in a scale assessing schizophrenic language disturbances. Psychol. Med. 29, 161-170.

Moritz, S., Mersmann, K., Jacobsen, D., Wilke, U., Andresen, B., Naber, D., 2001a. Further evidence for 'hyper-priming' in thought-disordered schizophrenics. Psychol. Med. 31, 221-229.

Moritz, S., Mersmann, K., Jacobsen, D., Kloss, M., Andresen, B., Pawlik, K., Naber, D., 2001b. Enhanced semantic priming in thought-disordered schizophrenic patients using a word pronunciation task. Schizophr. Res. 2-3, 303-308.

Moritz, S., Andresen, B., Jacobsen, D., Mersmann, K., Wilke, U., Lambert, M., Krausz, M., Naber, D., Neuropsychological correlates of schizophrenic syndromes in patients treated with atypical neuroleptics. Eur. Psychiatry, in press.

Neely, J.H., 1977. Semantic priming and retrieval from lexical memory: roles of inhibitionless spreading activation and limited-capacity attention. J. Exp. Psychol.: Gen. 106, 226-254.

Neely, J.H., 1991. Semantic priming effects in visual word recognition: a selective review of current findings and theories. In: Besner, D., Humphreys, G.W. (Eds.), Basic Progresses in Reading and Visual Word Recognition. Erlbaum, Hillsdale, NJ, pp. 264-333.

Ober, B.A., Vinogradov, S., Shenaut, G.K., 1995. Semantic priming of category relations in schizophrenia. Neuropsychology 9 , $220-228$.

Ober, B.A., Vinogradov, S., Shenaut, G.K., 1997. Automatic versus controlled semantic priming in schizophrenia. Neuropsychology $11,506-513$.

Salomé, F., Boyer, P., Fayol, M., 2000. The effects of psychoactive drugs and neuroleptics on language in normal subjects and schizophrenic patients: a review. Eur. Psychiatry 15, 461-469.

Spitzer, M., 1997. A cognitive neuroscience view of schizophrenic thought disorder. Schizophr. Bull. 23, 29-50.

Spitzer, M., Braun, U., Hermle, L., Maier, S., 1993a. Associative semantic network dysfunction in thought-disordered schizophrenic patients: direct evidence from indirect semantic priming. Biol. Psychiatry 34, 864-877.

Spitzer, M., Braun, U., Maier, S., Hermle, L., Maher, B.A., 1993b. Indirect semantic priming in schizophrenic patients. Schizophr. Res. 11, 71-80.

Spitzer, M., Weisker, I., Winter, M., Maier, S., Hermle, L., Maher, B.A., 1994. Semantic and phonological priming in schizophrenia. J. Abnorm. Psychol. 103, 485-494.

Vinogradov, S., Ober, B.A., Shenaut, G.K., 1992. Semantic priming of word pronunciation and lexical decision in schizophrenia. Schizophr. Res. 8, 171-181.

Weisbrod, M., Maier, S., Harig, S., Himmelsbach, U., Spitzer, M., 1998. Lateralised semantic and indirect semantic priming effects in people with schizophrenia. Br. J. Psychiatry 172, 142-146. 